transport tube. Nucleic acid extraction and real-time PCR was performed on the BD MAX instrument using the BioGX Mycoplasma-Ureaplasma reagent. In this analysis we evaluated only the detection of MG.

Results Genital samples from 416 women were tested using both the AMG and BioGX reagents. The overall agreement was $99.3 \%(\kappa=.876)$ with 402 negative and 11 positive samples in agreement. Two samples were positive only using the BioGX reagents and one specimen was only positive with AMG.

Conclusion In this MG detection study, we showed good performance of BioGX reagents on the BD MAX platform. The BioGX reagents can be used with any real-time PCR system, thus expanding diagnostic capacity to many laboratories. The performance in this study was very similar to that of AMG. Reagents capable of wider testing can facilitate epidemiologic studies designed to understand the impact of MG infection.

Disclosure No significant relationships.

\section{P605 TESTING AND TREATMENT STRATEGIES FOR LIMITING DRUG RESISTANCE IN MYCOPLASMA GENITALIUM}

${ }^{1}$ Peter White*, ${ }^{2}$ Ruthie Birger. ${ }^{1}$ Imperial College School of Public Health, MRC Centre for Global Infectious Disease Analysis and NIHR Health Protection Research Unit in Modelling Methodology, Department of Infectious Disease Epidemiology, London, UK; ${ }^{2}$ Yale School of Public Health, Department of Epidemiology of Microbial Diseases, New Haven, USA

\subsection{6/sextrans-2019-sti.673}

Background Mycoplasma genitalium ( $\mathrm{Mg}$ ) has rapidly increased its resistance to azithromycin, which has been first-line therapy for non-chlamydial-non-gonococcal urethritis (NCNGU), a proportion of which is due to $\mathrm{Mg}$, and for treating $\mathrm{Mg}$ specifically. New commercial nucleic acid amplification tests (NAATs) are likely to greatly increase diagnosis and treatment of $\mathrm{Mg}$, potentially promoting resistance. We previously developed the first transmission-dynamic model of $\mathrm{Mg}$, which we now use to examine alternative approaches to NAAT testing and treatment.

Methods Our model synthesises evidence from surveillance data, and epidemiological and behavioural studies, and accounts for parameter uncertainty, including the fitness-costs and benefits of drug resistance. The model incorporates resistance due to de novo mutation and transmission. We examined scenarios regarding (i)targeting of NAAT (only testing symptomatic patients and partners vs testing all patients); (ii)using NAATs detecting resistance vs only detecting $\mathrm{Mg}$; (iii)choice of first-line therapy (including continuing using azithromycin except where resistance has been detected vs alternative first-line regimen).

Results If azithromycin continues to be first-line therapy then resistance (and incidence of sequelae) will continue to rise, exacerbated by increased NAATs-based diagnoses. If asymptomatic screening occurs then resistance will increase 3.9(95\% CI:2.1-5.7) times as rapidly as if only symptomatic patients and partners are tested. Pre-treatment resistance testing mitigates but does not prevent increases in resistance, due to resistance arising from frequent de novo mutation. Long-term outcomes of alternative regimens are highly uncertain.

Conclusion This work supports recommendations not to screen for $\mathrm{Mg}$ until a better treatment regimen has been determined. NAATs should include resistance-testing but this is not a panacea. Improved understanding of Mg's natural history is urgently required, along with better surveillance of testing, diagnosis, and treatment, to monitor clinical adherence to guidelines, quantify drug-resistance fitness costs and benefits, and reduce uncertainty in decision-making. Internet-based testing and prescribing is a grave concern and needs to be controlled.

Disclosure No significant relationships.

\section{P606 OH MG! THE SYMPTOMS OF MYCOPLASMA GENITALIUM IN WOMEN}

${ }^{1}$ Rosie Latimer*, ${ }^{1}$ Lenka Vodstrcil, ${ }^{1}$ Tim Read, ${ }^{2}$ Michelle Doyle, ${ }^{2}$ Christopher Fairley,

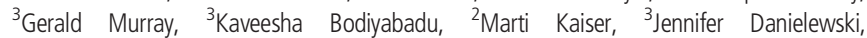
${ }^{4}$ Elisa Mokany, ${ }^{4}$ Litty Tan, ${ }^{2}$ Eric Chow, ${ }^{3}$ Suzanne Garland, ${ }^{2}$ Catriona Bradshaw. ${ }^{7}$ Monash University, Central Clinical School, Carlton, Australia; ${ }^{2}$ Alfred Health, Melbourne Sexual Health Centre, Carlton, Australia; ${ }^{3}$ The Royal Women's Hospital, Centre for Women's Infectious Disease Research, Parkville, Australia; ${ }^{4}$ SpeeDx Pty Ltd., Sydney, Australia

\subsection{6/sextrans-2019-sti.674}

Background While the contribution of Mycoplasma genitalium (MG) to symptoms in men is well described, less is known about its clinical presentation in women. Data support an association with cervicitis, but an association with pelvic inflammatory disease is contentious. We undertook a study of 1200 symptomatic and asymptomatic women to determine the prevalence of MG and macrolide resistance, and to determine its association with common genital symptoms in women to inform indications for testing.

Methods Women attending Melbourne Sexual Health Centre from 18th April 2017 (in progress) were tested for MG and macrolide resistance (ResistancePlusMG SpeeDx, Sydney), chlamydia and gonorrhoea (Aptima Combo 2, Hologic), trichomonasis (microscopy and culture), bacterial vaginosis (BV) and candida (microscopy). Women underwent examination and completed a questionnaire on symptoms. The prevalence of MG, macrolide-resistance, STIs and coinfection, and association with genital symptoms and signs, was determined by univariate and multivariable analysis.

Results Of 1054 women enrolled to date (968 symptomatic and 86 asymptomatic), 62 women (6\%, 95\%CI 5-7\%) tested positive for MG, with macrolide-resistance detected in 54\% (95\% CI 41-67\%). Chlamydia and gonorrhoea were detected in $8 \%(95 \% \mathrm{CI} 6-9 \%)$ and $1 \%(95 \% \mathrm{CI} 1-2 \%)$ of the 1054 women respectively. Of the 62 women infected with MG, $42 \%$ (95\%CI 30-55) also had BV, 26\% (95\%CI 16-38) candida, 6\% (95\%CI 2-16) chlamydia and 2\% (95\%CI 0-9) gonorrhoea. MG prevalence did not differ between symptomatic and asymptomatic women ( $6 \%$ vs $5 \%, \mathrm{p}=0.614)$. No specific genital symptoms or signs were significantly associated with $\mathrm{MG}$, in contrast to chlamydia, which was associated with post-coital bleeding (OR 1.7, $\mathrm{p}=0.04)$ and cervicitis (OR 2.3, $\mathrm{p}=0.014$ ).

Conclusion MG was as common as chlamydia in our clinic population but in contrast to chlamydia was not associated with any specific clinical features that would inform testing practices. Macrolide resistance was detected in half of cases and coinfection with BV was particularly common.

Disclosure No significant relationships. 